Methods: Disease activity data and blood samples from 40 patients with RA before and up to 12 months after starting a biologic therapy were collected. The study included patients starting intravenously administered Abatacept $(A B A ; n=10)$, Infliximab (INF;n=10), Tocilizumab (TCZ;n=10), and Rituximab (RTX;n=10), at approved dose regimens for RA treatment. HDLc-EC was measured on paired serum samples using THP-1 macrophages and a fluorometric assay for cholesterol measurement. ANOVA was used to compare paired continuous data, and Pearson' $r$ value was calculated for correlations.

Results: Disease activity assessed by DAS28-CRP and CDAl significantly dropped during all treatments. No significant changes in total and high or light density cholesterol fractions were detected. HDLc-EC at baseline was $22 \pm 3 \%$ with a statistically significant increase up to $25 \pm 3 \%$ and $27 \pm 4 \%$ after 6 and 12 months of treatment, respectively $(P<0.001)$ [Figure A]. Patients treated with INF and RTX demonstrated the higher rise in HDLC-EC, already after 6 months and lasting up to 12 months of treatment. ABA and TCZ treated patients after 6 months had a slight HDLc-EC rising, with a subsequent plateau. We observed an opposite correlation between HDLc-EC and disease activity by DAS28-CRP and CDAI $(r=-0.2 ; P=0.01)$ [Figure $B$ ]. Finally, no $\mathrm{CV}$ events were detected during the study follow-up.
A

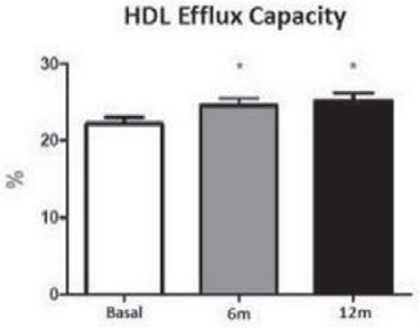

B

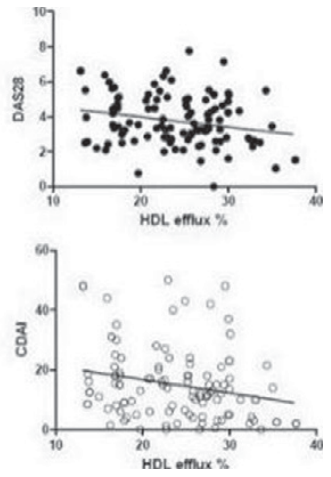

Conclusions: Biologic treatment can influence the HDLc-EC of RA patients, already after 6 months of therapy, and is associated with changes in disease activity and inflammatory status. Infliximab and Rituximab seem to have an enhanced impact on HDLC-EC. Future studies need to address the mechanisms beyond these intriguing findings.

References:

[1] Arts EE et al. Ann Rheum Dis. 2015;74:998-1003.

[2] Woodworth TG, den Broeder AA. Best Pract Res Clin Rheumatol. 2015;29:543-9.

[3] Rohatgi A. et al. N Engl J Med 2014;371:2383-93.

[4] Liao KP et al. J Am Heart Assoc. 2015;4(2).

[5] Ormseth MJ. et al. Arthritis Rheumatol. 2016;68:2099-105.

Disclosure of Interest: None declared

DOI: 10.1136/annrheumdis-2017-eular.2680

\section{THU0144 COGNITIVE FUNCTION OF PATIENTS WITH RHEUMATOID ARTHRITIS IS ASSOCIATED WITH DISEASE ACTIVITY BUT NOT CAROTID ATHEROSCLEROTIC CHANGES}

G.-T. Kim ${ }^{1}$, H.-S. Tag ${ }^{1}$, Y.-K. Kim ${ }^{1}$, S.-G. Lee ${ }^{2}$, E.-K. Park ${ }^{2}$, J.-H. Park ${ }^{2}$, J.-W. Lee ${ }^{3}$, S.-H. Kim ${ }^{4}$, J.-H. Lee ${ }^{5}$. ${ }^{1}$ Department of Internal Medicine, Kosin University College of Medicine; ${ }^{2}$ Department of Internal Medicine, Pusan National University Hospital; ${ }^{3}$ Department of Internal Medicine, Busan St. Mary's Hospital; ${ }^{4}$ Department of Internal Medicine, Inje University College of Medicine; ${ }^{5}$ Department of Internal Medicine, Maryknoll Medical Center, Busan, Korea, Republic Of

Background: Rheumatoid arthritis (RA) is a complex inflammatory disease that has features of atherosclerosis and cognitive decline. Although the relationship between atherosclerosis and cognitive impairment has been studied and replicated, whether cognitive deficits in RA can be attributed to their atherosclerotic changes is not well understood.

Objectives: This study investigated the cognitive function in patients with RA using the Korean version of the Consortium to Establish a Registry for Alzheimer's disease (CERAD-K) neuropsychological battery and evaluated whether cognitive function was affected by the carotid arterial atherosclerosis.

Methods: We examined seventy RA patients and forty healthy controls. RA activity was assessed by disease activity score with 28 joints-erythrocyte sedimentation rate (DAS28-ESR) and objective memory impairment was defined as a performance score of 1.5 standard deviations below the respective agespecific, education-specific, and sex-specific normative means for at least one of the four episodic memory tests in CERAD-K; the Word List Memory, Word List Recall, Word List Recognition, and Constructional Recall tests [1]. Carotid arteries were scanned for the presence of plaque and intima-media thickness (IMT). We assessed potential risk factors of cognitive impairment in RA patients using regression analyses.

Results: Of the CERAD-K subtests, there were a significant difference between the RA patients and healthy controls in verbal fluency $(12.97 \pm 3.73$ vs $15.48 \pm 4.57$, respectively; $p=0.004)$ and Boston Naming Test ( $11.50 \pm 2.08$ vs $12.30 \pm 1.77$, respectively; $\mathrm{p}=0.035)$. Carotid ultrasound revealed significantly more plaques in the RA patients than in the healthy controls (39\% vs $15 \%$, respectively; $p=0.017)$. RA patients with memory impairment have significantly higher score of DAS28-ESR (4.14 \pm 0.99 vs $2.60 \pm 0.88$, respectively; $p<0.001)$, age $(65.71 \pm 7.71$ vs $58.50 \pm 11.33$, respectively; $p=0.009)$, and mean cIMT $(0.56 \pm 0.10$ vs $0.50 \pm 0.08$, respectively; $p=0.027$ ) compared to $R A$ patients without memory impairment. In multivariable regression analysis, CERAD-K total score showed a significant negative correlation with age $(\beta=-0.415, p<0.001)$ or DAS28-ESR $(\beta=-4.685$, $p<0.001$ ), but no correlation was found between CERAD-K total score and presence of plaque or cIMT (Table 1).

Table 1. Regression analysis of mean CERAD-K total scores and clinical parameters

\begin{tabular}{lccccccc}
\hline & \multicolumn{9}{c}{ Univariable analysis } & \multicolumn{5}{c}{ Multivariable analysis } \\
\hline Total (n=70) & $\begin{array}{c}\text { Coefficient } \\
(\boldsymbol{\beta})\end{array}$ & $95 \% \mathrm{Cl}$ & $\mathbf{P}$ & $\mathbf{R}^{2}$ & $\begin{array}{c}\text { Coefficient } \\
(\boldsymbol{\beta})\end{array}$ & $95 \% \mathrm{Cl}$ & $\mathbf{P}$ \\
\hline Age (years) & -0.494 & $(-0.711,-0.277)$ & $<0.001$ & 0.232 & -0.415 & $(-0.607,-0.224)$ & $<0.001$ \\
Male (\%) & 2.121 & $(-5.079,9.320)$ & 0.559 & 0.005 & & & \\
RF (IU/MI) & 0.003 & $(-0.014,0.020)$ & 0.733 & 0.002 & & & \\
hSCRP (mmol/L) & -0.074 & $(-0.284,0.135)$ & 0.482 & 0.007 & & & \\
DAS28 & -5.402 & $(-7.552,-3.252)$ & $<0.001$ & 0.270 & -4.685 & $(-6.629,-2.742)$ & $<0.001$ \\
TC & 0.020 & $(-0.038,0.078)$ & 0.484 & 0.007 & & & \\
Mean cIMT & -26.082 & $(-56.267,4.103)$ & 0.089 & 0.042 & & & \\
Presence of plaque & -1.209 & $(-6.79,4.371)$ & 0.667 & 0.003 & & & \\
\hline RF: rheumatoid factor, hsCRP: high sensitivity C-reactive protein, DAS28: disease activity score 28, TC: total cholesterol, \\
clMT: carotid intima-media thickness
\end{tabular}

Conclusions: Our results indicate that disease activity of RA and aging contribute to cognitive impairment, but there was no association between cognitive function and clinical or subclinical carotid atherosclerotic changes in RA patients. References:

[1] Lee JH, Lee KU, Lee DY, Kim KW, Jhoo JH, Kim JH, et al. Development of the Korean version of the consortium to Establish a Registry for Alzheimer's disease Assessment Packet (CERAD-K): clinical and neuropsychological assessment batteries. J Gerontol B Psychol Sci Soc Sci 2002;57:47-53.

Disclosure of Interest: None declared

DOI: 10.1136/annrheumdis-2017-eular.3942

\section{THU0145 IMPACT OF DIFFERENT FORMULATIONS OF "PATIENT GLOBAL ASSESSMENT" ON REMISSION CLASSIFICATION BY DISEASE ACTIVITY INDICES IN RHEUMATOID ARTHRITIS}

G. Eugénio $^{1}$, R. Ferreira ${ }^{1,2}$, C. Silva ${ }^{3}$, C. Medeiros ${ }^{1}$, J.A.P. Silva ${ }^{1,3}$

C. Duarte ${ }^{1,3}$. ${ }^{1}$ CHUc-Huc; ${ }^{2}$ UICISA:E; ${ }^{3}$ FMUC, Coimbra, Portugal

Background: Patient global assessment (PGA) of disease activity is included in a large number of composite indices of disease activity and definitions of remission in Rheumatoid Arthritis (RA). However, the actual question is formulated in a variety of different ways according to the instrument considered.

Objectives: To evaluate how 6 different formulations of PGA affect patient estimates and impact upon disease activity and remission rates as assessed by 4 Disease Activity Indices.

Methods: Consecutive RA patients followed in a Rheumatology outpatient department were included in this cross-sectional study. Data collection comprised: 28 joint counts (tender and swollen), C-reactive protein (CRP) and 6 different PGA formulations. The chosen formulations were the ones stated in the: v1) Portuguese National Registry Reuma.pt, the locally used formulation; v2) ACR/EULAR provisional definition of remission (considered in this study as the "standard"); v3) CDAI and SDAI; v4) Disease Activity Score (DAS28) assessment of general health; v5) DAS28 assessment of disease activity (the currently used); v6) one, exploratory, developed by the investigators, including idiomatic cultural expressions. ACR/EULAR Boolean criteria, CDAI, SDAI, and DAS28-CRP (4v) were used to test how these 6 PGA formulations change the rates of remission. PGA differences were assessed by descriptive analyses (including patients with $P G A \leq 10$ and $\leq 20 \mathrm{~mm}$ ) and Bland-Altman test.

Results: In total, 193 patients were included (82\% female, mean (SD) age of 59 (13) years, mean disease duration of 12 (9) years and $31 \%$ under biologics). The average PGA ranged from 42.3 (25.3) to 48.1 (26.7) $\mathrm{mm}$ as measured in different formulations. The ACR/EULAR (v2) formulation yielded the largest proportion of

Table 1. Descriptive statistics of the 6 PGA's formulations ( $n=193$ )

\begin{tabular}{llll}
\hline PGA Formulation & Mean (SD) & \multicolumn{2}{c}{ PGA below cut-off $\mathrm{n}(\%)$} \\
\cline { 3 - 4 } & & $\leq 10 \mathrm{~mm}$ & $\leq 20 \mathrm{~mm}$ \\
\hline v1 Reuma.pt & $47.5(28.0)$ & $26(13.5)$ & $43(22.3)$ \\
V2 ACR/EULAR & $43.5(27.9)$ & $31(16.1)$ & $48(24.9)$ \\
V3 CDAI/SDAI & $47.2(25.9)$ & $23(11.9)$ & $34(17.6)$ \\
v4 DAS28-GH & $42.9(25.3)$ & $27(14.0)$ & $42(21.8)$ \\
v5 DAS28-DA & $42.3(25.3)$ & $28(14.5)$ & $44(22.8)$ \\
v6 Investigators & $48.1(26.7)$ & $22(11.4)$ & $35(18.1)$ \\
\hline
\end{tabular}

